\title{
Concepção do cuidador acerca do desenvolvimento da criança com paralisia cerebral
}

\section{após intervenção fisioterapêutica}

\author{
Caregiver conception about child development with cerebral palsy after intervention \\ physiotherapeutic
}

Concepción de cuidados sobre el desarrollo de niños con parálisis cerebral después de intervención

\section{fisioterapéutica}

\author{
Ariane Gomes da Silva \\ ORCID: https://orcid.org/0000-0002-9674-8077 \\ Universidade Estadual do Sudoeste da Bahia, Brasil \\ E-mail: ari-gomes@live.com \\ Isnara Teixeira de Britto \\ ORCID: https://orcid.org/0000-0001-7916-2085 \\ Universidade Estadual do Sudoeste da Bahia, Brasil \\ E-mail: isnara.britto@ hotmail.com \\ Maria Nice Dutra de Oliveira \\ ORCID: https://orcid.org/0000-0003-1362-9222 \\ Universidade Estadual do Sudoeste da Bahia, Brasil \\ E-mail: mnicedutra@gmail.com \\ Nayara Alves de Sousa \\ ORCID: https://orcid.org/0000-0003-4746-0103 \\ Universidade Estadual do Sudoeste da Bahia, Brasil \\ E-mail: nayara.sousa1@ @otmail.com \\ Robson dos Anjos Matos \\ ORCID: https://orcid.org/0000-0001-8923-3780 \\ Universidade Estadual do Sudoeste da Bahia, Brasil \\ E-mail: robson.matoos@hotmail.com \\ Elayny Lopes Costa \\ ORCID: http://orcid.org/0000-0002-7436-1170 \\ Universidade Estadual do Sudoeste da Bahia, Brasil \\ E-mail: elaynylopes@gmail.com
}

\begin{abstract}
Resumo
Esse artigo tem como objetivo conhecer a concepção do cuidador das crianças com paralisia cerebral, sobre a interferência do tratamento fisioterapêutico em seu desenvolvimento. Adota o caráter descritivo com abordagem qualitativa, desenvolvido na Clínica Escola de Fisioterapia (CEF) da Universidade Estadual do Sudoeste da Bahia. A coleta foi realizada nos domicílios dos cuidadores e/ou na própria CEF. Participaram desse estudo cuidadores de crianças com paralisia cerebral (PC) com idades entre 2 a 10 anos, que frequentaram, no mínimo 6 meses, a fisioterapia. Foi utilizada uma entrevista semiestruturada analisada por meio da análise de conteúdo de Bardin. O estudo atendeu os decretos da Resolução 466/12 aprovada pelo Plenário do Conselho Nacional de Saúde. Com a análise das entrevistas emergiram categorias como: o significado da fisioterapia para o cuidador, a criança após a fisioterapia e como estaria se não houvesse o tratamento, participação do cuidador no tratamento, a criança gostar de fazer a fisioterapia e as dificuldades encontradas para dar continuidade ao tratamento. Concluiu-se que, maioritariamente, os cuidadores consideram importante o tratamento fisioterapêutico para o desenvolvimento de suas crianças.
\end{abstract}

Palavras-chave: Cuidadores; Fisioterapia; Paralisia cerebral; Desenvolvimento infantil.

\begin{abstract}
This article aims to understand the conception of caregivers of children with cerebral palsy about the interference of physical therapy treatment in their development. It adopts a descriptive character with a qualitative approach, developed at the School of Physiotherapy Clinic (CEF) of the State University of Southwest Bahia. The collection was carried out in the caregivers' homes and/or in the CEF itself. Caregivers of children with cerebral palsy (CP) aged 2 to 10 years, who attended physical therapy for at least 6 months, participated in this study. It was a semi-structured interview analyzed using Bardin's content analysis. The study complied with the decrees of Resolution 466/12 approved by the Plenary of the National Health Council. With the analysis of changes, categories emerged such as: the meaning of physical therapy for the caregiver, a child after physical therapy and how it would be if there was no treatment, caregiver
\end{abstract}


participation in the treatment, a child enjoying physical therapy and the difficulties encountered in giving process to treatment. It was concluded that, for the most part, caregivers consider physical therapy treatment to be important for the development of their children.

Keywords: Caregivers; Physical therapy; Cerebral palsy; Child development.

\section{Resumen}

Este artículo tiene como objetivo comprender la concepción de los cuidadores de niños con parálisis cerebral sobre la interferencia del tratamiento de fisioterapia en su desarrollo. Adopta un carácter descriptivo con enfoque cualitativo, desarrollado en la Escuela Clínica de Fisioterapia (CEF) de la Universidad Estatal del Sudoeste de Bahía. La recogida se realizó en los domicilios de los cuidadores y / o en el propio CEF. Participaron en este estudio cuidadores de niños con parálisis cerebral (PC) de 2 a 10 años, que asistieron a fisioterapia durante al menos 6 meses. Fue una entrevista semiestructurada analizada utilizando el análisis de contenido de Bardin. El estudio cumplió con los decretos de la Resolución 466/12 aprobados por el Pleno del Consejo Nacional de Salud. Con el análisis de cambios surgieron categorías como: el significado de la fisioterapia para el cuidador, un niño después de la fisioterapia y cómo sería si no hubiera tratamiento, la participación del cuidador en el tratamiento, un niño disfrutando de la fisioterapia y las dificultades. encontrado al dar el proceso al tratamiento. Se concluyó que, en su mayor parte, los cuidadores consideran que el tratamiento de fisioterapia es importante para el desarrollo de sus hijos.

Palabras clave: Cuidadores; Fisioterapia; Parálisis cerebral; Desarrollo infantil.

\section{Introdução}

A encefalopatia crônica não progressiva na infância (ECNPI), comumente conhecida como paralisia cerebral (PC) foi pela primeira vez referida em 1862 por William John Little, quando este publicou um trabalho sobre a relação do parto complicado e prematuro com as alterações físicas e mentais nas crianças (Rézio \& Formiga, 2014). Apesar do termo ECNPI designar essa patologia em sua completude e especificidade, atualmente, a maioria dos estudos ainda utilizam o termo PC (Monteiro, Abreu \& Valenti, 2015).

A PC é um transtorno do neurodesenvolvimento que pode causar alterações do tônus, postura e movimento devido a lesões ou malformações no sistema nervoso central em crescimento antes, durante ou pós-parto (Evaristo \& Almeida, 2016). Essa enfermidade apresenta prevalência estimada média de 1,5 e 3,8 a cada 1000 nascidos em todo o mundo (Jm, Ee, Sg \& Ne, 2017).

É uma patologia heterogênea que pode ser classificada de acordo às suas manifestações clínicas, em: espástica, caracterizada pela hipertonia elástica, com predomínio em grupamento muscular flexor dos membros superiores e extensor em membros inferiores; discinética, caracterizada por movimentos involuntários amplos, posturas anormais em decorrência da incoordenação motora e tônus muscular variável; atáxica, caracterizada pela redução dos tônus muscular e desorientação espacial, devido à alteração cerebelar; hipotônica, apresentam reflexos miotáticos reduzidos e podem estar associados a déficit cognitivo e crises epilépticas; e mista quando apresentam duas ou mais formas de manifestação clínica (Monteiro et al., 2015). Há também a padronização quanto à função motora, sendo utilizado o Sistema de Classificação da Função Motora Grossa (GMFCS) que classifica o acometimento motor em cinco níveis Zanelato et al. (2014). Pacientes dos níveis I e II tem comprometimento motor leve e deambulam de forma independente; no nível III o comprometimento é moderado e usam dispositivos auxiliares para deambular; nos níveis IV e V tem comprometimento grave e precisam de cadeira de rodas para se deslocar (Ribeiro, Vandenberghe, Prudente \& Porto, 2016).

A PC impacta no desempenho diário dessas crianças que por vezes necessitam de auxílio para executar suas atividades da vida diária (AVD’S), esse cuidado é prestado, geralmente, por um familiar, assumindo o papel de cuidador que podem enfrentar maiores dificuldades no dia a dia (Domenech, Tavares, Ruedell \& Nobre 2016). Assim, é essencial o acompanhamento de uma equipe multidisciplinar, ratificando, deste modo, o papel da fisioterapia (Moraes, Souza, Caruso \& Machado, 2017). O tratamento fisioterapêutico busca melhorar o condicionamento biomecânico da criança a fim de permitir a manutenção da saúde e/ou a aquisição de autonomia, quando possível, para realizar suas AVD’S (Luna, Lucena, Farias \& Melo, 2018). 
Deste modo, constantemente surgem indagações aos profissionais fisioterapeutas quanto a efetividade das intervenções realizadas e com isso há vários instrumentos como o Sistema de Classificação da Função Motora Grossa - GMFCS e o Gross Motor Function Measurel Classificação da Função Motora Grossa (GMFM) que dá um feedback ao profissional quanto o prognostico e quantificação da função motora de crianças com PC (Gomes \& Golin, 2013), porém sabe-se que os cuidadores podem vivenciar alterações indetectáveis nestes instrumentos e ao mesmo tempo se eles identificam alterações positivas adquiridas com o tratamento, a adesão será muito maior e as atividades que forem prescritas para domicílio eles se esforçarão para executá-las, contribuindo desta forma para o melhor desenvolvimento da criança (Alpino, Valenciono, Furlaneto \& Zechim, 2013).

Neste saber, observa-se a relevância do estudo em identificar a concepção do cuidador de quanto e como o tratamento fisioterapêutico alterou no desenvolvimento da criança com PC. Dessa maneira, o presente estudo teve como objetivo conhecer a concepção do cuidador das crianças com paralisia cerebral, sobre a interferência do tratamento fisioterapêutico em seu desenvolvimento.

\section{Metodologia}

Trata-se de um estudo de caráter descritivo e abordagem qualitativa. A pesquisa qualitativa se baseia na compreensão do assunto de modo aprofundado (Aporta \& Lacerda, 2018).

O estudo foi desenvolvido com os cuidadores cadastrados na Clínica Escola de Fisioterapia (CEF) da Universidade Estadual do Sudoeste da Bahia (UESB), e presta atendimento exclusivamente ao Sistema Único de Saúde (SUS), com serviços em diversas áreas da fisioterapia, inclusive em neuropediatria e está localizada no município de Jequié/BA que possui uma população total de 151.895 habitantes Instituto Brasileiro de Geografia e Estatística [IBGE] (2010).

A coleta foi realizada no terceiro trimestre de 2019, nos domicílios dos cuidadores ou na própria CEF, dependendo da vontade e comodidade dos colaboradores. Sendo adotados como critérios de inclusão que os colaboradores fossem cuidadores de crianças com PC, com idades entre 2 a 10 anos, tendo em vista que essa faixa etária se insere na categoria criança de acordo com o Estatuto da Criança e do Adolescente [ECA] (2019). Portanto, foram inseridas aquelas que frequentaram regularmente a fisioterapia na CEF em um período mínimo de seis meses. Foram excluídos os que não atenderem a esses critérios ou que não aderiram à pesquisa.

Cada cuidador foi mencionado na ordem em que as entrevistas foram feitas e de acordo ao parentesco com a criança. Sendo nomeados por: M (para mãe), V (para avó), P (para pai), constituindo, assim, 3 mães (M1, M2, M3), 1 avó (V1) e 1 pai (P1), totalizando 5 cuidadores.

Para a coleta de dados foi utilizada a entrevista semiestruturada constituída de questões abertas e fechadas referentes aos dados sociodemográficos do cuidador e da criança; dados sobre o período gestacional e o parto da criança; e as perguntas, a segui, referentes ao tratamento fisioterapêutico: para o (a) senhor (a), qual o significado do papel da fisioterapia no tratamento de sua criança?; o que o (a) senhor (a) acha que a fisioterapia influenciou no desenvolvimento da sua criança?; quais as mudanças no desenvolvimento de sua criança o (a) senhor (a) relaciona ao tratamento fisioterapêutico?; como o (a) senhor (a) acha que estaria sua criança se não fizesse a fisioterapia?; fale o significado da fisioterapia na vida de sua criança; e faz outra terapia além do tratamento fisioterapêutico?. As entrevistas foram gravadas em instrumento (gravador de voz) para posterior transcrição e análise dos relatos.

A análise dos dados foi feita através da análise de conteúdo, seguindo as etapas propostas por Bardin. Essa técnica analisa o conteúdo das entrevistas, buscando relacionar estruturas semânticas com estruturas sociológicas dos enunciados, seguindo as suas características gerais: objetividade, sistematização e inferência (Hoffm, 2013). 
O estudo atendeu os decretos da Resolução 466/12, aprovada pelo Plenário do Conselho Nacional de Saúde, a qual foi aprovada pelo Comitê de Ética e Pesquisa da UESB, número do parecer: 3.461.248, em 18 de Julho de 2019. Sendo que os voluntários assinaram o Termo de Consentimento Livre e Esclarecido.

\section{Resultados e Discussão}

Antes de iniciar a coleta obteve-se um total de nove pacientes, cadastrados e atendidos na CEF que atendiam aos critérios de inclusão, dos quais cinco aceitaram participar.

As informações sociodemográficas dos cuidadores mostraram que a idade média foi de 37,2 anos $\pm 9,17$ anos. Ao estado civil 80\% tinham um (a) companheiro (a); 80\% eram do sexo feminino, sendo que destes $20 \%$ correspondia a avó e $60 \%$ as mães das crianças com PC.

Quando interrogados sobre sua ocupação, a cuidadora M2 (20\%) referiu ter sua renda de forma autônoma; M1 e V1 (40\%) referiram cuidar da criança; M3 (20\%) ser do lar e P1 (20\%) disse estar desempregado.

As crianças apresentaram uma média de 5,2 anos $\pm 2,99$ de atendimento, sendo o tempo menor de 1 ano e maior de 9 anos; média de 8,2 anos de idade $\pm 1,83$ e $80 \%$ eram do sexo feminino.

Quando questionados sobre a gestação da criança, todos disseram que a mãe fez o pré-natal, iniciando em pelo menos a $12^{\mathrm{a}}$ e $13^{\mathrm{a}}$ semana, realizando no mínimo 6 consultas. Dois cuidadores P1 e M2 referiram que a gestação da criança foi de risco, devido ao fato das gestantes serem hipertensas. No que diz respeito ao tipo de parto apenas M1 teve parto normal, os demais foram cesáreos.

Em relação a equipe que acompanha as crianças, M2 tem o acompanhamento fisioterapêutico, os demais disseram ter também outros profissionais envolvidos, como o fonoaudiólogo (P1, V1, M1, M3); equoterapeutas (M1, M3).

A seguir, apresentam-se os resultados referentes a análise das entrevistas transcritas, onde foi possível obter seis categorias temáticas que serão discutidas a seguir.

\section{Significado da Fisioterapia}

Ao investigar junto aos cuidadores acerca do significado da Fisioterapia ficou perceptível o quanto consideram importante o tratamento fisioterapêutico para as suas crianças.

“[... ] Pra gente o significado é ajudar ela no desenvolvimento dela [...] motor [...] porque justamente ela tem a paralisia ne [...] tem as limitações dela então a fisio ajuda muito, a fisioterapia ajuda muito, [...] a questão do alongamento, a questão dela ta se deslocando [...]” P1

“[...] Pra mim foi o melhor de todos [...] de todos os tratamentos é o melhor [...]” M1

"É muito bom ne não [...] a fisioterapia [...] a fisioterapia, eu acho que [...] fazendo a fisioterapia [...] se movimento melhor, sabe [...] quando tá fazendo a fisioterapia, entendeu [...]” M2

“[...] Aaah a fisioterapia para meu filho é muito importante porque estimula muito ele, entendeu [...] a musculação [...] e relaxa mais, entendeu [...]" M3

Outros estudos corroboram com as respostas supracitadas, onde ressaltaram que os cuidadores de crianças com PC perceberam a importância e benefícios do tratamento fisioterapêutico em seu desenvolvimento motor, psicológico e social. Do 
mesmo modo em que o acompanhamento fisioterapêutico se apresenta positivamente na melhora da qualidade de vida dos pacientes (Martins \& Souza, 2018) (Domenech et al., 2016).

\section{A criança após a Fisioterapia}

As crianças com quadro de PC apresentam limitações funcionais na execução das suas AVD'S devido ao seu quadro geral de redução de força, amplitude de movimento limitada, restrição articular e alterações do controle motor (Budtinger \& Muller, 2018). A fisioterapia influenciará na reabilitação ao criar possiblidades cotidianas que auxiliam no desenvolvimento de suas habilidades funcionais (Mockford \& Caulton, 2008). Neste enlace foi possível perceber, tendo em vista as falas abaixo, que os cuidadores conseguem observar esses avanços.

“[...] Por exemplo, se alimentar sozinha [...] assim alguns alimentos [...] alimentos mais sólidos, liquido ainda ela derrama assim mesmo [...] mas depois das fisios ela consegue pegar na colher já os alimentos mei sólidos [...] se alimenta sozinha, entendeu?! Não derrama tanto como antes [...] antes das fisios ave maria ela nem colocava a colher na boca [...] Ela consegue se locomover sozinha do jeito dela ne [...] as vezes rastando, as vezes ela tenta engatinhar e cai levanta, as vezes rasta sentada, as vezes rasta de barriga no chão, do jeito dela mas ela se locomove [...] para levantar que nem eu te falei se ela se apoiar nessa paredinha ai, ela levanta sozinha [...] agora ela não anda ainda $[\ldots] " P 1$

“[...] antes das fisios ave maria ela nem colocava a colher na boca [...] a coordenação dela, a parte na escola mesmo ta melhorando bastante depois das fisios [...] ela pegava no lápis com muita força, ainda mais que ela é canhota, pegava com muita força...rasgava o caderno [...] hoje em dia ela não rasga mais... depois das fisios ela melhorou bastante $[\ldots] ” P 1$

Diferentemente das citadas acima, a cuidadora V1, não observou nenhuma contribuição importante no desenvolvimento de sua neta, embora ela tente quantificar o ganho em sua fala. Um dos pressupostos clínicos revela que quanto maior o grau de comprometimento, maiores serão os fatores limitantes associados à funcionalidade (Dias, Freitas, Formiga \& Viana, 2010), deste modo, o objetivo, para além de outras instancia do tratamento fisioterapêutico, será a prevenção de agravos e deformidades (Gomes \& Golin, 2013).

As famílias de crianças com PC sofrem com a escassez de informações que lhes são passadas sobre a patologia e o processo de cuidar, esse déficit permeia o profissional de saúde, principalmente devido a cultura biomédica que verticaliza a informação e não estimula a comunicação, deste modo, o ganho ou manutenção de agravos e deformidades percebidas pelo profissional são evidentes aos seus olhos, porém não são perceptíveis sob o olhar do cuidador, se isso não lhes forem esclarecidos (Baltor, Borges \& Dupas, 2014).

“[...] Ai realmente assim [...] eu acho que ela [...] assim não éhh [...] achei que ta [...] ficou tudo normal mesmo que ela era $[\ldots] " V 1$

“[...] Melhorou assim né [...] algumas coisinhas ne [...] mas, vamos supor que ela desenvolveu assim uns $10 \%$ [...] assim no caso ne [...]. ela olha para as pessoas [...]. não é, fica olhando para as pessoas [...] ela fica se mexendo assim $[\ldots]$ tudo isso $[. .$.$] ". V 1$ 
As demais cuidadoras identificaram os avanços alcançados, principalmente no que diz respeito ao seu desenvolvimento motor. Estes achados se assemelham ao que foi encontrado na literatura, onde demonstram a significância do atendimento fisioterapêutico na melhora da aquisição motora de crianças com PC (Palacio, Ferdinande \& Gnoatto, 2008).

“[...] ela começou a sentar [...] ai começou a passar de deitado pra sentado sozinha, demorou um tempinho também, ela ficava sentada só com uma sustentação [...] alguma coisinha atrás [...] mas foi muita coisa que eu achei que melhorou" M1

"éh porque que sem a fisioterapia ela ta sem o equilíbrio no pescocinho [...] ela já ne [...]. ela consegue com a fisioterapia [...]" M2

“[...] é bom a fisioterapia. [...] porque as mãozinhas dela ne [...] que depois de ir pra UTI ela perdeu os movimentos no bracinho esquerdo [...] ela quase não movimenta eles ne [...] eles trabalham assim com as mãozinhas dela pra ela ficar abrindo a mão [...]" M3

Nesse aspecto observou-se que os cuidadores identificam evolução nas crianças, no entanto ainda é frágil a sua percepção quanto ao cuidado fisioterapêutico, devido ao provável déficit de informação que lhes são passados sobre o tratamento e como isto beneficiará sua criança. Isto corrobora com o que foi apresentado em estudo de Mello, Ichisato e Marcon (2012), no qual eles atribuem esse fato ao déficit de comunicação entre o profissional e o cuidador, reforçando também que quando bem estabelecido essa interação, a reabilitação do paciente pediátrico com PC torna-se mais promissora.

\section{Se não houvesse a Fisioterapia}

As abordagens terapêuticas para o tratamento da PC são diversas e objetiva estimular a melhora das habilidades motoras, o aprimoramento da função motora grossa, prevenção de contraturas e deformidades ( Q. \& C., 2016) e mostra-se eficaz na redução da espasticidade (Ey \& Wh, 2017). Através das entrevistas ficou perceptível que os cuidadores perceberam o quão ruim seria para suas crianças se não houvesse o tratamento fisioterapêutico.

“[...] AAAAH ia ta que nem muitos falaram ne, quando viram ela [...] falou: -essa menina vai vegetar- [...] então assim, ela não ia ta tão avançada como tá hoje, sem as fisios [...] sem as fisios tava complicado viu [...] ela só queria ficar mais deitada [...] primeiro que se ficar parado vai atrofiar mais ainda [...]” P1

“[...] Estaria mais parada, sem se mexer [...]” V1

“[...] Eu acho que se não tivesse feito a fisioterapia [...] ela taria bem mais atrasada do que ela ta hoje [...]” M1

“[...] Num sei [...] porque a fisioterapia é bom [...] porque eu deixo minha filha mais assim ne[...] deitada ne, e com a fisioterapia ela tem mais movimento [...]" M2

“[...] Aaah acho que ele tava acamado, e muito molinho, não tinha coordenação motora entendeu [...] eu acho que ele tava acamado do mesmo jeito que nasceu [...] M3

Os cuidadores percebem que se faz necessário o atendimento fisioterapêutico, observados a partir das suas presunções que se não fosse feito haveria atraso no desenvolvimento dessas crianças. Desta forma, em estudo de Luna et al. (2018), 
reforçaram essas atribuições do tratamento, em que objetivam não somente a busca do máximo de autonomia como também a prevenção de complicações.

\section{Participação do cuidador no tratamento}

Os cuidadores foram questionados sobre a continuidade do tratamento em suas residências, verificou-se que a maioria deles realizavam as orientações passadas pelos profissionais ou reproduziam em casa o que observavam no atendimento.

“[... A A todo momento, hoje mesmo a gente deu foi risada fazendo fisioterapia dentro de casa ne ... eu exercitando ela [...] tudo que é passado, que eu aprendo com vocês la, conhecimento, eu passo pra ela aqui, eu falo mesmo [...] alongo, alongo devargarzinho ai eu boto o extensor e tal ai boto pra ela andar, calço o tênis dela, falo com ela, dou uma volta na varanda segurando ela [...] andando [...] pra não ficar parada [...] é um pouquinho mais ajuda [...]” P1

“[...] Assim o que [...] o pessoal fazia [...] fazia aqui ne [...]” V1

“[...] Sempre que estamos em casa, a gente reforça o que eles fazem la [...]” M1

“[...] Eu num [...] além de ter muita coisa pra fazer, as vezes to atarefada, mas também quando eu tenho tempo entendeu [...] eu conserto as mãozinhas dela... faço com que ela se movimente [...]” M2

A atuação dos cuidadores no tratamento se faz necessário para que haja continuidade em seu lar, de modo que, quando as orientações não são passadas restringe a possibilidade de mais avanços no desenvolvimento da criança com PC, isso se justifica que na presença das orientações, há o incentivo diário que torna a criança mais motivada (Gração \& Santos, 2008). Outro aspecto é que fortalece o vínculo de confiança entre o cuidador e o fisioterapeuta e o vínculo afetivo entre o cuidador e a criança, sendo que através das orientações pode-se potencializar os resultados alcançados no atendimento (Souza \& Knobel, 2019).

\section{A criança gosta de fazer a fisioterapia}

A cuidadora M2 ressaltou a felicidade de sua criança em ir para a fisioterapia. Em estudo de Carvalho, Rodrigues, Silva \& Oliveira (2010), eles relacionam essa satisfação à convivência social da criança com outras que possuem alterações semelhantes, deste modo, elas se sentem parte de um grupo.

“[...] quando acorda ela pra fisioterapia [...] ela fala '-mãe, [...] vai pra pia, vai pra pia [...] ela fica muito feliz, entendeu [...] ela gosta de ir pra fisioterapia [...] segunda feira mesmo eu arrumada com ela e ela ai se balançando na cadeira falando que ia pra pi. pia...sabe [...]” M2

\section{Dificuldades encontradas para dar continuidade ao tratamento}

Durante a entrevista mesmo não sendo questionado sobre, o cuidador P1 falou sobre sua dificuldade para dar continuidade ao tratamento. Essa dificuldade foi a questão da rotatividade do estágio na CEF devido a mudança de profissionais, de modo que quando a criança se acostuma com o estagiário que o atende, acontece a mudança dos mesmos.

“[...] eu só acho uma pena vocês lá não ter total liberdade de avançar mais porquê[...] e assim [...] é bom em uns pontos e em outro que eu acho ruim na [...] é a questão do rodizio [...] eu sei que vocês, como estudante tem que passar, tem que ter aquele rodizio, mas é complicado, porque quando a criança tá se adaptando com você 
[...] vem outro e depois vem outro e tem outro lá que é bom também [...] que as vezes você tem uma visão [...] ai tem outra visão, ai melhora mais ainda [...]" P1

A fala do cuidador P1 condiz com o citado em estudo de Subtil, Goes, Gomes e Souza (2011), no qual o processo de adaptação e evolução no desenvolvimento do paciente pode estar relacionado com o relacionamento interpessoal e cuidado integral deste com o fisioterapeuta, principalmente para melhor adesão ao tratamento.

\section{“[...] eu gosto da fisioterapia [...] só que o que me pega é o transporte pra vim me pegar aqui ne [...]” M2}

A fala supracitada corrobora com a dificuldade evidenciada em estudo Dantas, Pontes, Assis e Collet (2012), em que relatam o problema diário do cuidador em relação a escassez de recursos financeiros e o deslocamento da criança até o local de atendimento, por isso necessitam de suporte e apoio social que o auxiliem no transporte.

\section{Conclusão}

Nesta pesquisa verificou-se que, maioritariamente, os cuidadores consideram a importância do tratamento fisioterapêutico para o desenvolvimento das aquisições motoras de suas crianças. Contudo, ainda é frágil a percepção dos ganhos pela dificuldade de comunicação entre o profissional e o cuidador.

O estudo também destaca pontos positivos como o convívio social das crianças no serviço e a disposição dos cuidadores para seguir as orientações no domicílio. Quanto aos pontos negativos, diz respeito à acessibilidade para dar continuidade ao tratamento e a rotatividade de profissionais em decorrência do estágio.

Deste modo, o estudo mostrou-se relevante ao conhecer sob a ótica do cuidador o desenvolvimento da criança com PC após intervenção fisioterapêutica, além de reconhecer e reforçar que o profissional deve estar sempre atento em estabelecer uma melhor interação com o cuidador, promovendo melhor qualidade de vida desta criança através de sua família. Recomenda-se que novos estudos sejam realizados com um número maior de cuidadores e crianças com maior tempo de intervenção fisioterapêutica.

\section{Referências}

Alpino, Â. M. S., Valenciono, P. J., Furlaneto, B. B. \& Zechim, F. C. (2013). Orientações de Fisioterapia a Mães de Adolescentes com Paralisia Cerebral: Abordagem Educativa para o Cuidar. Rev. Bras. Ed. Esp., 19(4), 597-610

Aporta, a. P., \& Lacerda, C. B. F. de. (2018). Estudo de Caso sobre Atividades Desenvolvidas para um Aluno com Autismo no Ensino Fundamental I. Revista Brasileira de Educação Especial, 24(1), 45-58.

Baltor, M. R. R., Borges, A. A., \& Dupas, G. (2014). Interação com a criança com paralisia cerebral: comunicação e estigma. Esc Anna Nery, $18(1)$, 47-53.

Budtinger, L. F., \& Muller, A. B. (2018). Método PediasuitTM no tratamento da paralisia cerebral: relato de casos. Revista FisiSenectus, 6(1), 4-12.

Carvalho, J. T. de M., Rodrigues, N. M., Silva, L. V. C. da, \& Oliveira, D. A. de. (2010). Qualidade de da das mães de crianças e adolescentes com paralisia cerebral. Fisioter. Mov., 23(3), 389-397.

Dantas, M. S. de A., Pontes, J. F., Assis, W. D. de, \& Collet, N. (2012). Facilidades e dificuldades da família no cuidado à criança com paralisia cerebral. Rev. Gaúcha Enferm, 33(31), 73-80.

Dias, A. C. B., Freitas, J. C., Formiga, C. K. M. R., \& Viana, F. P. (2010). Desempenho funcional de crianças com paralisia cerebral participantes de tratamento multidisciplinar. Fisioter. Pesqui., 17(3), 225-229.

Domenech, A. C. P., Tavares, K. O., Ruedell, A. M., \& Nobre, J. R. da S. (2016). Cerebral palsy: the meaning of physical therapy for mother caregivers. Fisioter Mov, 29(4), 757-765. 
ECA. (2019). Lei no 8.069, de 13 de julho de 1990.Evaristo, F. L., \& Almeida, M. A. (2016). Benefícios do Programa PECS-Adaptado para um Aluno com Paralisia Cerebral. Rev. Bras. Educ. Espec, 22(4), 543-558.

Ey, P., \& Wh, K. (2017). Effect of neurodevelopmental treatment-based physical therapy on the change of muscle strength, spasticity, and gross motor function in children with spastic cerebral palsy. J Phys Ther Sci. 29(6), 966-969.

Gomes, C. de O., \& Golin, M. O. (2013). Tratamento Fisioterapêutico Na Paralisia Cerebral Tetraparesia Espástica, Segundo Conceito Bobath. Rev Neurocienc, 21(2), 278-285.

Gomes, C. R. A., Araújo, I. F. de, \& Maciel, S. C. (2014). Avaliaçao da funçao motora grossa pela GMFM pré e pós cirurgia ortopédica de membros inferiores em pacientes com paralisia cerebral. Acta Fisiátr., 21(1), 16-20.

Gração, D. C., \& Santos, M. G. M. (2008). A percepção materna sobre a paralisia cerebral no cenário da orientação familiar. Fisioter. Mov., 21(2), 107-113.

Hoffm, R. C. (2013). Análise de conteúdo: da teoria à prática em pesquisas sociais aplicadas às organizações. Revista Interinstitucional de Psicologia, 6(2), $179-191$.

Instituto Brasileiro de Geografia e Estatística (IBGE). (2020)

Jm, R., Ee, C., Sg, N., \& Ne, O. C. (2017). Exercise interventions for cerebral palsy (Review). Cochrane Database Of Systematic Reviews (Online).

Luna, M. M. de A., Lucena, P. L. de, Farias, A. E. M. de, \& Melo, V. F. C. de. (2018). O acompanhamento fisioterapêutico de crianças com sequelas de paralisia cerebral atendidas no NASF do município de Alagoa Nova. Rev. Pesqui. Cuid. Fundam. (Online), 10(3), 70-73.

Martins, P. V., \& Souza, S. M. de. (2018). Paralisia cerebral: itinerário terapêutico na perspectiva do cuidador familiar e a importância da fisioterapia. Revista Eletrônica Estácio Saúde, 8(1), 22-28.

Mello, R., Ichisato, S. M. T., \& Marcon, S. S. (2012). Percepção da família quanto à doença e ao cuidado fisioterapêutico de pessoas com paralisia cerebral. Rev. Bras. Enferm., 65(1), 104-109.

Mockford, M., \& Caulton, J. (2008). Systematic review of progressive strength training in children and adolescents with cerebral palsy. Pediatr Phys Ther, 20(4), 318-333.

Monteiro, C. B. D. M., Abreu, L. C. de, \& Valenti, V. E. (2015). Paralisia Cerebral: Teoria e Prática. Plêiade

Moraes, M. C. de, Souza, H. L., Caruso, M. F. B., \& Machado, M. R. (2017). Benefícios do uso da realidade virtual no tratamento fisioterapêutico em pediatria. Revista de Trabalhos Acadêmicos-Universo Juiz de Fora, 1(4), 1-12.

Palacio, S. G., Ferdinande, A. K. S., \& Gnoatto, F. C. (2008). Análise do desempenho motor de uma criança com hemiparesia espástica pré e pós-tratamento fisioterapêutico: estudo de caso. Ciênc. Cuid. Saúde, 7(1), 127-131.

Q, C., Antonio, J., \& C., N. (2016). Efecto de la frecuencia e intensidad de intervenciones terapéuticas basadas en neurodesarrollo en niños con parálisis cerebral: una revisión sistemática. Rehabil. Integral (Impr.), 11(2), 99-107.

Rézio, G. S., \& Formiga, C. K. M. R. (2014). Inclusion of children with cerebral palsy in basic education. Fisioter Pesq., 21(1), 40-46.

Ribeiro, M. F. M., Vandenberghe, L., Prudente, C. O. M., \& Porto, C. C. (2016). Paralisia cerebral: faixa etária e gravidade do comprometimento do filho modificam o estresse e o enfrentamento materno. Ciência \& Saúde Coletiva, 21(10), 3203-3212.

Souza, J. S. de, \& Knobel, K. A. B. (2019). Guia ilustrado de orientações a cuidadores de crianças com deficiências neuromotoras. ConScientiae Saúde, 18(1), $8-17$.

Subtil, M. M. L., Goes, D. C., Gomes, T. C., \& Souza, M. L. de. (2011). O relacionamento interpessoal e a adesão na fisioterapia. Fisioter Mov., 24(4), 745753.

Zanelato, N., Rosanova, G. C. L., Santucc, F., Fragoso, D. da S., Campos, D., Narumia, L. C., \& Ozu, M. H. U. (2014). Associação dos níveis de GMFCS com grau de escolaridade, tipo de marcha, empregabilidade e dor em adultos com paralisia cerebral. Acta Fisiatr, 21(4), 158-161. 